\title{
Electrical stimulation for neuromuscular testing and training: state-of-the art and unresolved issues
}

\author{
Nicola A. Maffiuletti • Marco A. Minetto • \\ Dario Farina $\cdot$ Roberto Bottinelli
}

Received: 2 August 2011/Accepted: 15 August 2011/Published online: 25 August 2011

(C) Springer-Verlag 2011

\section{Introduction}

Contrary to other widespread forms of electrical stimulation, such as transcutaneous electrical nerve stimulation (TENS) and functional electrical stimulation (FES), neuromuscular electrical stimulation (NMES) is generally delivered to the muscle in static conditions (without functional movement occurring) and at sufficiently high current intensities to evoke visible muscle contractions (beyond motor threshold). NMES has received increasing attention in the last few years, because it has the potential to serve as:

- a strength training tool for healthy subjects and athletes, since its chronic use may induce neuromuscular adaptations similar/complementary to voluntary strength training;

Communicated by Susan A. Ward.

This article is published as part of the Special Issue Cluster on the XVIII Congress of the International Society of Electrophysiology and Kinesiology (ISEK 2010) that took place in Aalborg, Denmark on 16-19 June 2010.

N. A. Maffiuletti ( $\square)$

Neuromuscular Research Laboratory, Schulthess Clinic,

Lengghalde 2, 8008 Zurich, Switzerland

e-mail: nicola.maffiuletti@kws.ch

M. A. Minetto

Division of Endocrinology, Diabetology and Metabolism, Department of Internal Medicine, University of Turin, Turin, Italy

M. A. Minetto

Laboratory for Engineering of the Neuromuscular System

(LISiN), Department of Electronics, Politecnico di Torino,

Turin, Italy
- a rehabilitation and preventive tool for partially- or totally immobilized patients, since its chronic use may preserve muscle mass and function during prolonged periods of reduced muscular use;

- a testing tool for evaluating the neural and/or muscular function in vivo, since it is able to induce standardized muscle contractions whose electrical (EMG) and mechanical (torque) properties could be easily quantified; and

- a post-exercise recovery tool for athletes, since its acute application may increase muscle blood flow and therefore metabolite washout which could in turn accelerate recovery kinetics during and after exercise.

Portable NMES units are widely available to the general population. However, commercial claims regarding NMES use often go far beyond the existing scientific evidence. Moreover, due to the lack of general consensus in the scientific community about the main physiological and methodological features of NMES, end users are faced with confusion regarding its usage and effectiveness, so that they often prefer not to apply NMES or to apply it with exaggerated caution.

D. Farina

Department of Neurorehabilitation Engineering, Bernstein Center for Computational Neuroscience, University Medical Center Göttingen, Georg-August University, Göttingen, Germany

R. Bottinelli

Department of Physiology and Interuniversity Institute

of Myology, University of Pavia, Pavia, Italy

R. Bottinelli

Fondazione Salvatore Maugeri (IRCCS), Scientific Institute of Pavia, Pavia, Italy 
This cluster of articles is dedicated to selected topics relative to the physiology, methodology and applications of NMES (Fig. 1), with a state-of-the art scientific review on motor unit recruitment, acute muscular effects, chronic neural adaptations induced by NMES training, optimization of the stimulation paradigm to increase NMES effectiveness, and NMES use as a tool for strength training, neuromuscular testing and post-exercise recovery. The rationale behind the cluster is that, in order to be able to improve/optimize neuromuscular function by means of NMES (applications in Fig. 1), we need first to improve our knowledge on NMES (physiology in Fig. 1), and then improve the actual use of NMES (methodology in Fig. 1). This series of article is therefore addressed to practitioners and researchers from different fields (exercise training, exercise physiology, sport medicine, and even general medicine) whose main interest is the optimization of skeletal muscle function through NMES. After an overview of the group of articles, a series of unresolved issues and recommendations for future NMES research are presented.

\section{NMES physiology}

\section{Motor unit recruitment is peculiar}

Bickel et al. (2011) reviewed the issue of motor unit recruitment order during electrical stimulation. In contrast to the size-related orderly recruitment behaviour that has been documented for voluntary contractions, the accepted view is that nerve stimulation imposes a reversal of the motor unit activation order since large-diameter axons of the fast units are more easily excited by imposed electric fields than are small-diameter axons of the slow units. However, in the case of in vivo over-the-muscle stimulation (such as for NMES), the variable spatial distribution of the motor axonal branches in a non-uniform current field (current density decreases with increasing depth) has much greater importance than the excitability threshold (that is, the size) of the axonal branches, thus implying that motor unit recruitment is non-selective or random: motor units are activated without obvious sequencing related to unit type or size during NMES (i.e., "disorderly" recruitment). In addition, the peculiar motor unit recruitment imposed by electrical stimulation is superficial, spatially fixed, and incomplete (Gregory and Bickel 2005). The spatially fixed recruitment implies that the same motor units are repeatedly activated by the same amount of electrical current which, in turn, hastens the onset of muscle fatigue (as also discussed by Gondin et al. 2011b). Such early occurrence of fatigue represents a major limitation of NMES, and could eventually play a role in NMES-induced muscle damage (Nosaka et al. 2011). In order to maximize the spatial recruitment during NMES, thus minimizing the extent of muscle fatigue, it has been recommended to implement different expedients during a treatment session such as progressive increase in current intensity, alteration in muscle length, and displacement of active electrodes (Maffiuletti 2010). In this cluster of articles, further approaches have been proposed to alleviate, at least in part, discomfort and other possible complications associated to the electrically evoked contractions (Botter et al. 2011; Bergquist et al. 2011; Gobbo et al. 2011).

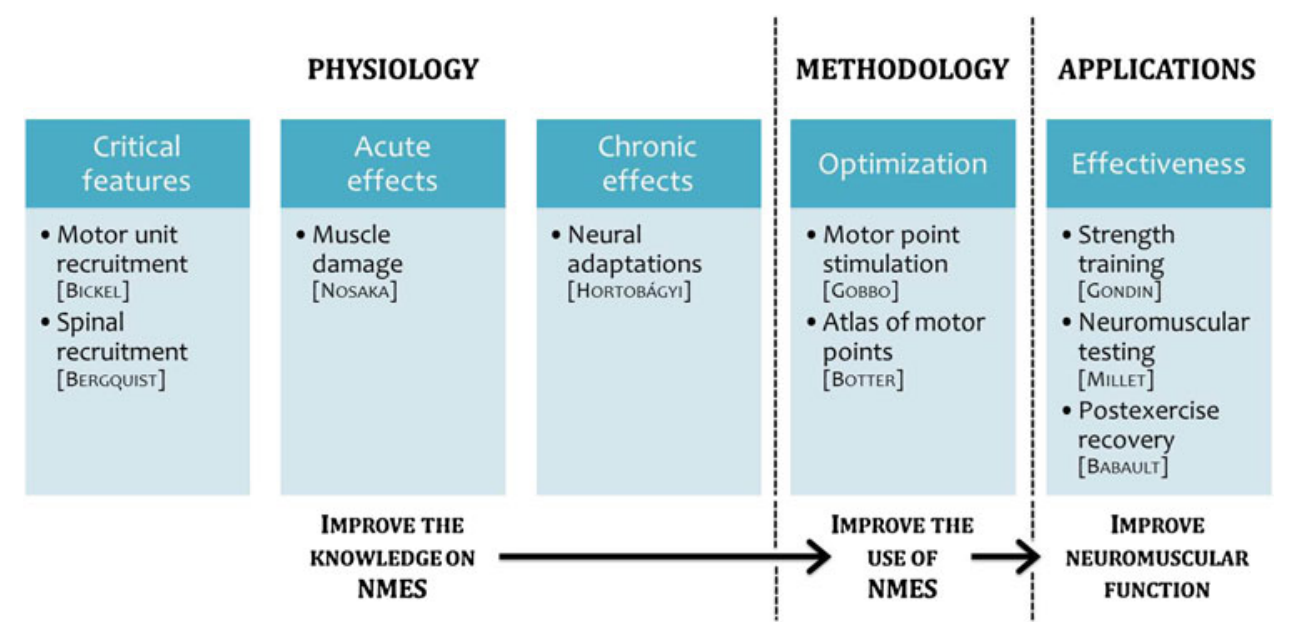

Fig. 1 Schematic representation of the rationale behind the cluster of articles (to be interpreted from left to right). Improving our knowledge on NMES physiological features and effects (physiology) is required before optimizing the use of NMES in the clinic (methodology). These two logical steps should necessarily precede all evidence-based applications of NMES directed toward the improvement of neuromuscular function in vivo. Within each column, studies are grouped by topic area, with the name of the first author presented in square brackets 
Bergquist et al. (2011) provided an overview of how peripheral (direct activation of motor axon branches) and central (reflexive recruitment of spinal motor neurons by the electrically evoked afferent volley) pathways contribute to electrically evoked contractions and suggested that some of the limitations of NMES (particularly discomfort and random recruitment) could be minimized by increasing the contribution through central pathways. In fact, motor unit recruitment through central pathways may be more orderly, less synchronous and more spatially diffuse throughout the muscle, than recruitment through purely peripheral pathways. Enhancing central recruitment during NMES requires the stimulation to be delivered at low pulse amplitudes (to minimize the antidromic block, that is the collision between the action potentials travelling antidromically along motor axons and those generated following the reflexive recruitment of motor neurons), pulse durations in the range $0.2-1 \mathrm{~ms}$ (to maximize the excitation of afferent axons that present a longer strength-duration time constant and lower rheobase than motor axons), stimulation train durations shorter than $2 \mathrm{~s}$ for stimulation over the nerve and longer than $2 \mathrm{~s}$ for stimulation over the muscle, and high $(100 \mathrm{~Hz})$ pulse frequencies (to increase the rate at which the sensory volley is sent to the spinal cord and supra-spinal centres). Based on these characteristics of the stimulation burst, this stimulation paradigm has been referred to as "wide-pulse high-frequency NMES".

Acute NMES use may cause profound muscle damage

Nosaka et al. (2011) reviewed the most recent evidence available for the muscle damage induced by NMES-evoked contractions, which resembles the type of damage produced by voluntary eccentric contractions, resulting in decreased maximal voluntary contraction (MVC) strength, increased circulating levels of muscle proteins and delayed onset muscle soreness. Muscle damage profile following NMES-evoked isometric contractions of the knee extensors is similar between different stimulation paradigms (pulsed vs. alternating current) for similar force outputs. Moreover, its magnitude is reduced following a second session of NMES performed two weeks after a first session: this phenomenon, known as "repeated bout effect", has originally been described for voluntary eccentric contractions and could be useful to limit the damage associated to NMES if the training or rehabilitation program includes "conditioning" sessions.

Strength training by NMES leads to considerable neural adaptations

Hortobágyi and Maffiuletti (2011) reviewed the changes in the function of the central nervous system (neural adaptations) that may occur with NMES strength training, which include spinal and supraspinal mechanisms of adaptation. Interestingly, the increases in MVC strength induced by NMES training seem to be mainly mediated by supraspinal rather than spinal changes, although no training study has yet documented the former adaptations. The fact that NMES and voluntary strength training may have different supraspinal effects in the contralateral homologous muscle should be used as a key point motivating the combination of the two forms of exercise in the context of neuromuscular retraining. Somatosensory and nociceptive inputs associated to NMES use may lead to changes in motor cortical excitability, which in turn can cause functional improvements to occur. Moreover NMES seems to be able to modify the excitability of interhemispheric connections and possibly the balance between interhemispheric excitation and inhibition. These facts lead us to the conjecture that NMES training can be viewed as a "neural" rather than a "muscular" treatment technique, particularly for neurological patients.

\section{NMES methodology}

Motor point stimulation as a sine qua non-condition to optimize NMES use

Gobbo et al. (2011) provided convincing evidence on the importance of motor point determination to minimize current intensity and discomfort, and to maximize the muscular tension evoked by NMES. They also used near infrared spectroscopy, which is a technique particularly suitable for investigating local metabolic changes of stimulated muscles (Muthalib et al. 2009), and were able to demonstrate that, besides contractile activity, muscle oxygen consumption and hyperaemia were significantly increased when motor point location was carefully determined with a pen electrode rather than being inferred from several legally marketed motor point charts. We recommend that the procedure described by Gobbo et al. (2011), which is completed in less than a minute, should be consistently incorporated in both clinical and research contexts to optimize NMES application.

Botter et al. (2011) investigated the uniformity of the muscle motor point location in the lower limb of healthy subjects and found different motor points innervating different portions of the quadriceps, posterior thigh, and tibialis anterior muscles. On this basis, they suggested that a maximization of the spatial recruitment during NMES could also be obtained through a multi-channel stimulation technique that involves a non-synchronous activation of different muscle volumes. Interestingly, it has recently been demonstrated that asynchronous low-frequency 
$(16 \mathrm{~Hz})$ stimulation of the quadriceps muscle using a multi-pad electrode (four channels) can elicit a strong, fused contraction, though producing less muscle fatigue compared to single-channel high-frequency $(30 \mathrm{~Hz})$ stimulation (Malesević et al. 2010).

\section{NMES applications}

NMES for optimizing muscle function: is that really useful?

Gondin et al. (2011b) provided an overview of the main training studies in which NMES has been applied to healthy subjects or even to competitive athletes with the objective to improve muscle function. As already pointed out in a recent review published in this journal (Maffiuletti 2010), the so-called training intensity (i.e., the level of force evoked by NMES, expressed as a fraction of the MVC force) seems to be the main determinant of NMES training effectiveness. In other words, muscles should produce the greatest relative tension when stimulated by NMES in order to maximize the training-induced strength gains (Lai et al. 1988; Selkowitz 1985). Interestingly, we plotted the MVC strength gains collected from different NMES training studies completed in our laboratory against respective training intensities, and confirmed that a doseresponse relation does exist between these two variables (Maffiuletti NA, unpublished observations). This would imply that the improvement in MVC strength induced by NMES training could be predicted from the average training intensity; for example, a training intensity of $40 \%$ MVC being predicted to improve MVC strength by $20 \%$. Such predictive equation may even be improved (and eventually extended to unhealthy populations) by adding the initial MVC strength and NMES training volume as independent variables.

Millet et al. (2011) presented the potential interests and fundamentals of electrical stimulation for the evaluation of voluntary activation, muscle contractility (with and without fatigue), as well as respiratory muscle function. Contrary to MVC assessment, contractions evoked by electrical stimulation are not or little influenced by motivational factors and can be easily standardized; however, many researchers still adopt electrical stimulation in a simplistic way, with scarce or no attention to physiological and methodological issues that may invalidate the test. The heterogeneity of electrical stimulation procedures already discussed for NMES training also applies to the evaluation of neuromuscular features, as different laboratories adopt different stimulation units, current characteristics, electrode arrangements, etc. We believe the increasing use of magnetic stimulation would necessarily reduce such heterogeneity even though, as discussed by Millet et al. (2011), the maximal power output of modern magnetic stimulators does not seem appropriate enough for subjects with excess subcutaneous adipose tissue.

Babault et al. (2011) reviewed the most recent evidence available for the effectiveness of non-tetanic low-intensity NMES as a post-exercise recovery tool. The idea that this exercise modality may increase blood flow thus favouring metabolite washout is appealing and at least in part conceivable. It is however hard to imagine how this would accelerate the time course of post-exercise recovery in sportsmen, as muscle recruitment with electrical stimulation is extremely limited and superficial. Moreover, none of the most common recovery strategies (such as massage, active exercise and stretching) have proved effective in accelerating recovery kinetics in sportsmen (Barnett 2006), so perhaps the question we should ask ourselves is "Aren't we wasting our time?". This is a clear example of how commercial claims (http://www.shopcompex.com/testimon ials) often go beyond scientific evidence. Regarding the management of exercise-induced pain and psychological benefits for recovery, additional evidence should be provided before considering NMES as a valid tool for postexercise recovery.

\section{Unresolved physiological and clinical issues, and future directions}

The purpose of this section is to outline elements of confusion and controversy that still plague the use of NMES as a testing and training modality in healthy and pathological subjects, and to examine some limitations in our understanding of NMES effects. With such an approach, we aim to provide a conceptual foundation for subsequent research studies in this area, but also for further implementation of NMES in the clinic. The same logical progression outlined in Fig. 1 is followed (from physiology to methodology to applications).

\section{Critical features}

The "wide-pulse high-frequency NMES" modality remains to be explored more in depth: for example, it is presently not defined whether inter-individual variability exists for the generation of the electrically elicited "extra" force that results from the reflexive recruitment of motor neurons and could also be amplified by an involuntary descending drive (Frigon et al. 2011). In addition, it has never been demonstrated that a stimulation paradigm aimed to produce a reflexive recruitment of motor neurons rather than to maximize the muscle tension can trigger positive neural and/or muscular adaptations favourable to muscle performance. 
As soon as the effectiveness of reflexive motor neuron recruitment can be demonstrated in controlled physiological studies, clinical investigations will be required to assess the feasibility of this new stimulation paradigm in clinical settings.

\section{Acute effects}

Mackey et al. (2011) recently showed that NMES-induced damage of the human medial gastrocnemius muscle results in de-adhesive, disassembly, and disorganization responses in the contractile connective tissue, followed by a delayed anabolic response leading to extracellular matrix remodelling. Besides these responses, the other mechanisms underlying NMES-induced muscle damage remain to be clarified. In particular, it remains to be established whether the damage provoked by NMES is specific for this type of stimulus or whether it resembles that produced by lengthening contractions, which represents the traditional experimental model for the study of muscle damage and regeneration. In addition, it remains to be elucidated whether the training effects of NMES on muscle and neural plasticity are reduced when muscle damage is minimized. In fact, it may be hypothesized that some damage is necessary to maximize muscle hypertrophy and strength gains that could be achieved by NMES.

Guarascio et al. (2004) reported a case of rhabdomyolysis in a young male subject presenting severe asthenia and increased levels of circulating muscle proteins induced by excessive NMES use. Even if routine measurements of circulating muscle proteins such as creatine kinase and myoglobin are not required during NMES training programs, it should be recommended to perform pre-training measurements and assessment of thyroid and kidney function (to rule out, at least in pathological subjects, the existence of an overt/subclinical hypothyroidism and renal insufficiency that may increase the risk of rhabdomyolysis) so as to facilitate the evaluation of subsequent muscle complaints. In addition, special attention is required if individuals subjected to NMES are under therapy with drugs that may produce muscle complaints (i.e., statins) or in the case of particular patient populations (e.g., critically ill patients). Finally, all subjects performing repeated sessions of NMES should be warned to stop the training and to contact their physician if they become symptomatic for severe muscle weakness and myalgias and notice dark discoloration of their urine.

\section{Chronic effects}

Strength training by NMES does promote neural and muscular adaptations that are complementary to the well-known effects of voluntary resistance training (Vanderthommen and Duchateau 2007). Future efforts should be directed at investigating more precisely the locus/loci of neural adaptations induced by NMES; in particular, supraspinal mechanisms/sites such as cortical excitability and cortical maps could be explored using transcranial magnetic stimulation and functional magnetic resonance imaging, techniques that are nowadays relatively accessible. The determinants of phenotypic variability in response to NMES strength training also require greater attention (Gondin et al. 2011a), with particular emphasis on factors that should be controlled, such as training intensity. Once the physiological mechanisms underlying neural and/or muscular changes to NMES training are substantiated by research evidence, one or both forms of adaptation (or even specific mechanisms) could be specifically targeted according to the individual needs of the end user. This would necessarily result in a more scientific and profitable use of NMES in clinical settings.

\section{Optimization}

The two main limitations associated to the use of NMES are the considerable discomfort and the relatively incomplete muscle recruitment, both imposed by the electrical field. Alternative techniques, stratagems and tools that might be able to minimize the impact of these limitations on NMES use should receive more attention. For example, the use of "distributed" NMES (Malesević et al. 2010), multi-path stimulation with large electrodes (Feil et al. 2011), and magnetic stimulation (Bustamante et al. 2010) should be encouraged as opposed to the classical NMES set-up, particularly for commonly stimulated large muscles. Additionally, a world-wide consensus on standardization of NMES terminology, which should include current and contraction characteristics, is considered necessary to allow a more uniform use of NMES both in research and in clinical settings.

\section{Effectiveness}

With regard to strength training, although NMES was originally introduced to treat muscle atrophy consequent to immobilization or denervation (Jackson and Seddon 1945), its use has traditionally been confined to exercise physiology, particularly for research purposes, while the clinical use of NMES has been relatively limited. As a result of this, the origin (neural vs. muscular) and the time course of adaptations to NMES strength training have been extensively investigated in healthy but not in pathological populations. It is now time to fill this gap! The experience gained from exercise physiology studies should now be redirected to pathological applications; good examples 
come from cardiorespiratory and internal care medicine where chronic obstructive pulmonary disease patients and critically ill patients are increasingly stimulated to preserve their muscle mass and function (Vivodtzev et al. 2008; Routsi et al. 2010). Additional efforts are needed to identify the most relevant applications of NMES training in the clinical setting, and also to discern its effectiveness and the time-course of neuromuscular adaptations for specific patient populations.

As far as muscle testing is concerned, future studies using in vitro stimulation patterns that are closer to the in vivo situation are required to further validate the existing methodologies (such as the twitch interpolation technique, see Place et al. 2008) or to conceive new ones. It would also be worth validating a quick, simple, standardized, and comprehensive testing procedure for the assessment of in vivo neuromuscular function in clinical settings, whose parameters could be easily manipulated according to patient characteristics and prefixed goals. This would provide a clear picture of the central (muscle activation) and peripheral (muscle contractility) components of muscle function, and certainly minimize the heterogeneity of in vivo neuromuscular evaluation between different centres.

Regarding post-exercise recovery optimization, the focus of future research studies should definitely be on acute physiological effects of non-tetanic low-intensity NMES (e.g., increased blood flow and metabolic alterations) rather than on global physical outcomes. Since there is no scientific evidence that this form of NMES could provide some physiological benefits for sportsmen, its application cannot currently be recommended to enhance post-exercise recovery, at least from a physiological perspective.

Acknowledgments The work reported in this article was supported by bank foundation "Compagnia di San Paolo" (Project "Neuromuscular Investigation and Conditioning in Endocrine Myopathy") (MAM), by the ERC grant DEMOVE and the Bernstein Focus Neurotechnology Göttingen (DF), and by MYOAGE Grant HEALTH-F2-2009-223576 ("Understanding and combating agerelated muscle weakness") (RB). The authors are grateful to Prof. R. Merletti (LISiN, Politecnico di Torino, Italy) for his careful review of the final version of the manuscript.

\section{References}

Babault N, Cometti C, Maffiuletti NA, Deley G (2011) Does electrical stimulation enhance post-exercise performance recovery? Eur J Appl Physiol. doi:10.1007/s00421-011-2117-7

Barnett A (2006) Using recovery modalities between training sessions in elite athletes: does it help? Sports Med 36:781-796

Bergquist AJ, Clair JM, Lagerquist O, Mang CS, Okuma Y, Collins DF (2011) Neuromuscular electrical stimulation: implications of the electrically-evoked sensory volley. Eur J Appl Physiol. doi: 10.1007/s00421-011-2087-9
Bickel SC, Gregory CM, Dean JC (2011) Motor unit recruitment during neuromuscular electrical stimulation: a critical appraisal. Eur J Appl Physiol (in press)

Botter A, Oprandi G, Lanfranco F, Allasia S, Maffiuletti NA, Minetto MA (2011) Atlas of the muscle motor points for the lower limb: implications for electrical stimulation procedures and electrode positioning. Eur J Appl Physiol. doi:10.1007/s00421-011-2093-y

Bustamante V, Lopez de Santa Maria E, Gorostiza A, Jimenez U, Galdiz JB (2010) Muscle training with repetitive magnetic stimulation of the quadriceps in severe COPD patients. Respir Med 104:237-245

Feil S, Newell J, Minogue C, Paessler HH (2011) The effectiveness of supplementing a standard rehabilitation program with superimposed neuromuscular electrical stimulation after anterior cruciate ligament reconstruction: a prospective, randomized, single-blind study. Am J Sports Med 39:1238-1247

Frigon A, Thompson CK, Johnson MD, Manuel M, Hornby TG, Heckman CJ (2011) Extra forces evoked during electrical stimulation of the muscle or its nerve are generated and modulated by a length-dependent intrinsic property of muscle in humans and cats. J Neurosci 31:5579-5588

Gobbo M, Gaffurini P, Bissolotti L, Esposito F, Orizio C (2011) Trascutaneous neuromuscular electrical stimulation: influence of electrode positioning and stimulus amplitude settings on muscle response. Eur J Appl Physiol. doi:10.1007/s00421-011-2047-4

Gondin J, Brocca L, Bellinzona E, D’Antona G, Maffiuletti NA, Miotti D, Pellegrino MA, Bottinelli R (2011a) Neuromuscular electrical stimulation training induces atypical adaptations of the human skeletal muscle phenotype: a functional and proteomic analysis. J Appl Physiol 110:433-450

Gondin J, Cozzone PJ, Bendahan D (2011b) Is high frequency neuromuscular electrical stimulation a suitable tool for muscle performance improvement in both healthy humans and athletes? Eur J Appl Physiol. doi:10.1007/s00421-011-2101-2

Gregory CM, Bickel CS (2005) Recruitment patterns in human skeletal muscle during electrical stimulation. Phys Ther 85:358-364

Guarascio P, Lusi EA, Soccorsi F (2004) Electronic muscular stimulators: a novel unsuspected cause of rhabdomyolysis. Br J Sports Med 38:505

Hortobágyi T, Maffiuletti NA (2011) Neural adaptations to electrical stimulation strength training. Eur J Appl Physiol. doi: 10.1007/s00421-011-2012-2

Jackson EC, Seddon HJ (1945) Galvanism and denervated muscle atrophy. Br Med J 2:485-486

Lai HS, De Domenico G, Strauss GR (1988) The effect of different electro-motor stimulation training intensities on strength improvement. Aust J Physiother 34:151-164

Mackey AL, Brandstetter S, Schjerling P, Bojsen-Moller J, Qvortrup $\mathrm{K}$, Pedersen MM, Doessing S, Kjaer M, Magnusson SP, Langberg H (2011) Sequenced response of extracellular matrix deadhesion and fibrotic regulators after muscle damage is involved in protection against future injury in human skeletal muscle. FASEB J 25:1943-1959

Maffiuletti NA (2010) Physiological and methodological considerations for the use of neuromuscular electrical stimulation. Eur J Appl Physiol 110:223-234

Malesević NM, Popović LZ, Schwirtlich L, Popović DB (2010) Distributed low-frequency functional electrical stimulation delays muscle fatigue compared to conventional stimulation. Muscle Nerve 42:556-562

Millet GY, Martin V, Martin A, Vergès S (2011) Electrical stimulation for testing neuromuscular function: from sport to pathology. Eur J Appl Physiol. doi:10.1007/s00421-011-1996-y

Muthalib M, Jubeau M, Millet GY, Maffiuletti NA, Nosaka K (2009) Comparison between electrically evoked and voluntary isometric 
contractions for biceps brachii muscle oxidative metabolism using near-infrared spectroscopy. Eur J Appl Physiol 107: $235-241$

Nosaka K, Aldayel A, Jubeau M, Chen TC (2011) Muscle damage induced by electrical stimulation. Eur J Appl Physiol. doi: 10.1007/s004021-011-2086-x

Place N, Yamada T, Bruton JD, Westerblad H (2008) Interpolated twitches in fatiguing single mouse muscle fibres: implications for the assessment of central fatigue. J Physiol 586:2799-2805

Routsi C, Gerovasili V, Vasileiadis I, Karatzanos E, Pitsolis T, Tripodaki E, Markaki V, Zervakis D, Nanas S (2010) Electrical muscle stimulation prevents critical illness polyneuromyopathy: a randomized parallel intervention trial. Crit Care 14:R74

Selkowitz DM (1985) Improvement in isometric strength of the quadriceps femoris muscle after training with electrical stimulation. Phys Ther 65:186-196

Vanderthommen M, Duchateau J (2007) Electrical stimulation as a modality to improve performance of the neuromuscular system. Exerc Sport Sci Rev 35:180-185

Vivodtzev I, Lacasse Y, Maltais F (2008) Neuromuscular electrical stimulation of the lower limbs in patients with chronic obstructive pulmonary disease. J Cardiopulm Rehabil Prev 28:79-91 